\title{
Molecular Electronics: A Review of Experimental Results
}

\author{
A. Erbe And S. Verleger \\ Universität Konstanz, FB Physik \\ Universitätsstr. 10, D-78457 Konstanz, Germany
}

\begin{abstract}
Molecular electronics aims for scaling down electronics to its ultimate limits by choosing single molecules as the building blocks of active devices. The advantages of this approach are the high reproducibility of molecular synthesis on the nanometer scale, the ability of molecules to form large structures by self-assembly, and the huge versatility of molecular complexes. On the other hand, conventional contacting techniques cannot form contacts on the single molecule scale and imaging techniques nowadays cannot provide a detailed image of such junctions. Therefore, the fabrication has to rely to some degree on self-organization of the constituents. The proof that a molecule has been contacted successfully can only be given by indirect methods, for example by measuring the current transport through the junctions. Here we give an overview of various techniques that were used successfully to contact molecules and to characterize them electrically. The techniques range from methods to contact single molecules to such which can be used to characterize ensembles of molecules. Especially, the comparison between such different techniques shows that a single measurement is always prone to artefacts originating from the unknown microscopic details of the junctions. It is therefore necessary to perform a statistically relevant number of measurements in order to resolve molecular properties. Various properties of the molecules can be studied. Special examples are the influence of conformational changes of the molecules, differences between various coupling endgroups of the molecules and effects of light-irradiation onto the molecular junctions.
\end{abstract}

PACS numbers: 85.65.+h, 73.63.-b, 85.35.-p

\section{Introduction}

The number of transistors in an integrated circuit grows exponentially, approximately doubling every two years. This behavior was predicted by Moore in 1965 and is since then known as "Moore's law" [1]. Such an increase in number of components requires, in turn, to scale down typical component sizes at a comparable rate. It is clear that classical electronic components cannot be scaled down without reaching any limits, because quantum mechanical effects will dominate the behavior of the elements if typical length scales will be of the same order as the Fermi wavelength of electrons (100 nm for typical 2D semiconductors and down to $1 \AA$ for metals). Gate oxides, which are less than 1-2 nm thick, are also no longer reliable due to uncontrollable surface roughness. Due to such reasons, new ways to build electronic devices have to be developed on the nanoscale. One of these ways is the use of single molecules as components of electronic circuits. The advantages of this approach are very clear: The molecular length scale is the smallest scale which can be imagined for entities carrying electric current. Molecules can be produced reproducibly in large numbers by chemical reactions. On the other hand, organic chemistry can generate a huge number of different molecules, thus creating a "molecular toolbox", which can be put together for larger scale electronic circuits. Molecular recognition can then be used to build these circuits in a process called self-assembly.

The use of single molecules as active electronic devices was already suggested in 1974 in a famous publication by Aviram and Ratner [2]. They proposed that a molecule with an electronic system that is divided into two parts, which are separated by a tunnel barrier, could act as a rectifying diode. At that time, however, fabrication methods were much too crude to produce structures on the nanometer scale, which would be needed to contact single molecules. It therefore took more than 20 years until the first successful measurements of molecular conductivity were reported [3-6]. In the years following these initial discoveries, an enormous amount of experimental results were presented using a large variety of contacting techniques. An excellent review of the development during these years is given in [7]. On the other hand, it also became clear that reproducible measurements on the nanometer scale pose extremely difficult challenges. Some of the effects which were attributed to molecular behavior turned out to be artifacts arising from uncontrollable variations of the configuration on the nanometer scale [8]. The experience that even the most carefully performed experiments are prone to such misinterpretations led to a first disillusion and the whole field was said to hit an early "mid-life crisis" [9]. The understanding of these first experiments made further developments towards more reliable contacting methods possible. Nowadays, there are a number of contacting techniques available, which have shown reproducible results on various molecules. In the following, we want to give an overview on some of these new results.

\section{Transport mechanisms}

Depending on the molecular structure, the coupling of the metal to the molecules, and the position of the 
Fermi energies of the metals with respect to the molecular energy levels, several transport mechanisms through the molecular backbone can be expected. In the simplest case, no free electronic states on the molecular orbitals are available for the electron coming from the metal, and the electron will tunnel through the distance between the two electrodes. If the electron can occupy some states on the molecule, the transport mechanism will sensitively depend on the coupling between the metal and the molecule. If this coupling is good, the coupling between various sites of the molecule will be the defining bottleneck for the transport and activated hopping between these sites will be the transport mechanism in this situation. On the other hand, if the coupling is poor, the metal-molecule junction can be regarded as a tunneling barrier and the molecule will be charged electron by electron. The transport in this regime is generally called the Coulomb blockade regime. In the following, we would like to discuss these transport mechanisms and describe the various $I-V$ characteristics, which arise from the different regimes.

\subsection{Tunneling}

When no electronic states on the molecule can be accessed, the electron needs to tunnel through the distance between the two contacting electrodes. In this scenario the height of the tunnel barrier is given by the offset $E_{\mathrm{a}}$ between the Fermi energy of the metal $E_{\mathrm{F}}$ and the closest molecular orbital, which can be either the highest occupied molecular orbital (HOMO) or the lowest unoccupied molecular orbital (LUMO). Typical energy differences are of the order of $2 \mathrm{eV}$, for example for gold contacts to simple alkane chains, where the the HOMO-LUMO gap is about $8 \mathrm{eV}$ and the position of $E_{\mathrm{F}}$ is determined by the work function of gold $(5 \mathrm{eV})$.

Tunneling is a stochastic process and therefore only depends on the height and width of the tunneling barrier and the rate at which the tunneling particle attempts to transfer. One of the most important consequences is that the conductance of a pure tunneling junction is temperature independent. This fact therefore represents one of the most straightforward tests to verify tunneling as the dominating transport mechanism. The dependence of the current $I$ on the applied voltage $V$ is determined by the amplitude of $V$. If $V$ is small compared to $E_{\mathrm{a}}$ it determines only the energy difference between the two electrodes. Therefore $I$ in this regime depends linearly on $V$. This dependence can be verified for measurements of $I-V$ characteristics between two gold electrodes, which are spaced by a few nanometers. The linear dependence is shown in Fig. 1.

If the applied bias $V$ exceeds a certain percentage of the barrier height, the barrier starts to be deformed. This leads to a deviation from the purely linear behavior and can already be seen in Fig. 1 at $V>5 \mathrm{~V}$. The theoretical description of the tunneling current is then given by the so-called Fowler-Nordheim equation

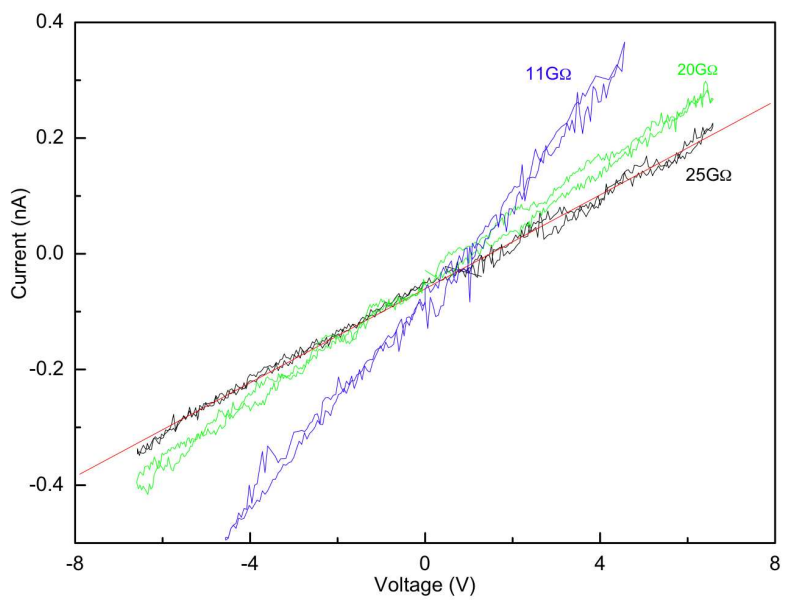

Fig. 1. Tunneling current between two gold tips situated a few nm apart from each other. The $I-V$ characteristics is linear, different resistance values correspond to different distances. After [34].

$$
I(V) \propto V^{2} \exp \left(-\frac{4 d \sqrt{2 m} \Phi^{3 / 2}}{3 e \hbar V}\right),
$$

where $d$ is the distance between the electrodes, $\Phi$ - the potential barrier, $m$ - the effective electron mass and $e$ - the electron charge. In both regimes the current depends exponentially on the distance between the electrodes.

\subsection{Activated transport}

In a molecule that is connected to metal electrodes with linker groups which provide a good electrical coupling the metallic and molecular orbitals can hybridize. In this scenario the overall conductance of the molecular junction is determined by the conductance along the molecular backbone. In the easiest case this would be given by a single molecular orbital, the whole molecule would simply act as a wire. If the coupling is weaker, transport is given by hopping of electrons from one metallic contact to the electronic states of the molecule and to the second contact. This hopping is an activated transport. The activation can be given either by thermal activation or by an increase in bias voltage. In both cases the transport depends exponentially on $V$ or $T$, respectively. The typical energy scale for these $\left(E_{\text {act }}\right)$ is determined by the coupling between the metallic and molecular states and their respective energies. This transport mechanism typically results in a so-called s-shaped curve, because the conductance is decreased around zero in a range which is comparable to $E_{\text {act }}$. An example of such a curve is shown in Fig. 2b.

\subsection{Coulomb blockade}

If a molecule is weakly coupled to the metallic electrodes, but the electrons can find electronic states within the molecular backbone, the whole junction can act as a capacitor. One plate of the capacitor is then given by 


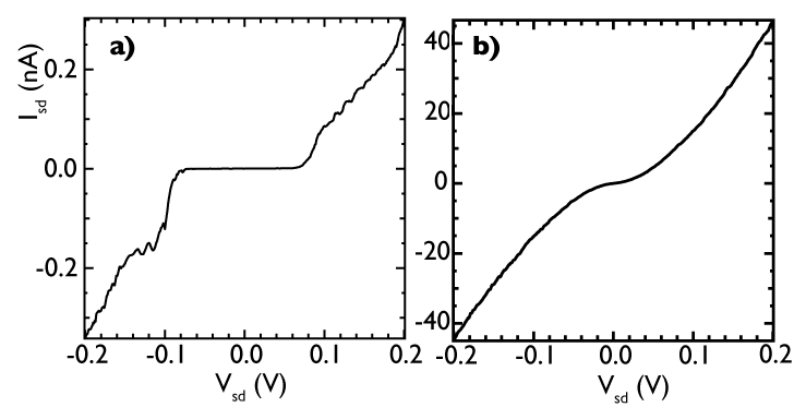

Fig. 2. Typical $I-V$ characteristics measured on polythiophene molecules at $4.5 \mathrm{~K}$. (a) Coulomb blockade; (b) activated transport.

the contacting electrode, the other by the molecule. It is well known that the energy to charge a capacitor with a charge $q$ is given by $E_{\mathrm{C}}=q^{2} / 2 C$. Scaling down the capacitors reduces the capacitance $C$ and thus increases the charging energy $E_{\mathrm{C}}$. Therefore the situation can arise that the energy $\left(k_{\mathrm{B}} T\right)$ which is thermally provided to the electrons is much smaller than $E_{\mathrm{C}}$ for a single electron $\left(e^{2} / 2 C\right)$. In this situation the capacitor cannot be charged and current through the molecule is blocked. Because this blockade is caused by the Coulomb repulsion of the electrodes it is generally known as the Coulomb blockade (CB).

In order to drive electronic current through the molecule additional energy must be provided. This can be done by increasing the bias voltage $V$ until $\mathrm{eV}>E_{\mathrm{C}}$. Then current through the molecule can occur by successively transporting single electrons through the structure. If the bias is increased further the number of conducting electrons is increased one by one and the current increases in a stepwise manner. A typical example for $\mathrm{CB}$ at low temperatures is shown in Fig. 2a. CB is temperature dependent, because these steps can be "washed out" by thermally activated electrons if the temperature is increased.

\subsection{External influences}

For the construction of molecular electronic devices it is mandatory that the electronic properties of the molecules can be controlled by means of external influences. One typical example is the addition of a third electrode (gate electrode), which can be used to tune the electrostatic energy of the molecular states. A structure showing CB effects can be used as a so-called single electron transistor (SET), when the gate electrode switches from one state in which the molecule can exchange electrons with the leads, to a state in which this transport is blocked [10]. A molecule can be affected by a gate in a very similar fashion, if the coupling to the leads is weak. The electronic system of strongly coupled molecules can be affected by changing the charge distribution, for example in functionalized side groups (see Fig. 3b).

Changes in temperature affect the overall conductance of molecules depending on the transport mechanism as

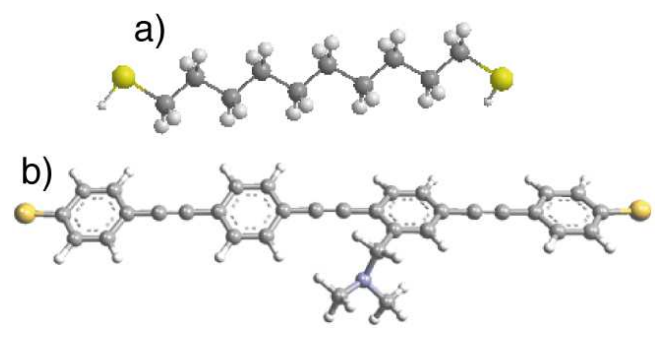

Fig. 3. (a) Non-conjugated alkane $\left(\mathrm{C}_{12}\right)$. (b) Conjugated molecule with functionalized side groups (dimethylaminomethyl-bis-mercapto- tolanyl-ethyne, DMA-BMTE).

mentioned in the previous sections. The influence of lattice vibrations (phonons) can only be seen at low temperatures, because thermal activation of phonons otherwise leads to thermal broadening. These resonances can be seen as maxima in the conductance and give insight into the internal degrees of freedom of the participating molecule.

\section{Design of molecules}

The various conduction schemes can be realized in molecules of different design. In general, the molecular structure can be split into two parts, the main central part and the end groups, which couple to the metallic electrodes. The whole system has to be regarded as an entity, therefore changes in one part of the structure (electrode, end group or central part of the molecule) will always influence the overall behavior. The central parts can be grouped into conjugated and non-conjugated molecules, depending on the overlap of the $\pi$-orbitals of the binding electrons. A typical non-conjugated molecule is an alkane chain, which can be produced in various lengths (12 C atoms in a chain are depicted in Fig. 3a).

The atoms in this molecule are connected via $\sigma$-bonds, therefore delocalization of electrons throughout the molecule cannot take place. It can be expected that this molecule acts as a tunnel barrier when it is situated between two electrodes. This assumption is confirmed by measurements of alkane chains of various lengths [11]. In contrast to this the carbon atoms in the molecule shown in Fig. $3 \mathrm{~b}$ are connected by $\pi$-orbitals. The overlap between such orbitals allows the electrons to be delocalized throughout the structure. In this case the electronic state of the whole molecule can be charged as an entity and transport through the molecule is expected to happen by activated hopping [12] for short molecules (shorter than $3 \mathrm{~nm}$ ). In longer molecules the interactions between the electrons and phonons can lead to the formation of polarons and therefore polaronic hopping can be the dominant transport mechanism [13].

In order to anchor the molecules to metallic electrodes functionalized end groups need to be incorporated, which couple to the molecular backbone electrically and provide mechanical and electrical stability to the electrodes. The 
most common end groups are thiol (SH) endgroups. It is well known that short molecules with thiol end groups form self-assembled monolayers (SAMs) on flat gold substrates [14] and GaAs [15] due to the interaction of the sulfur with the surface atoms. Thiol groups on both ends of the molecule can provide coupling to two closely spaced electrodes. This scheme has been used for many experiments on molecular electronics. More recently, other end groups have been studied in order to improve the reliability of the electronic coupling between molecules and metal $[16]$.

\section{Contacting techniques}
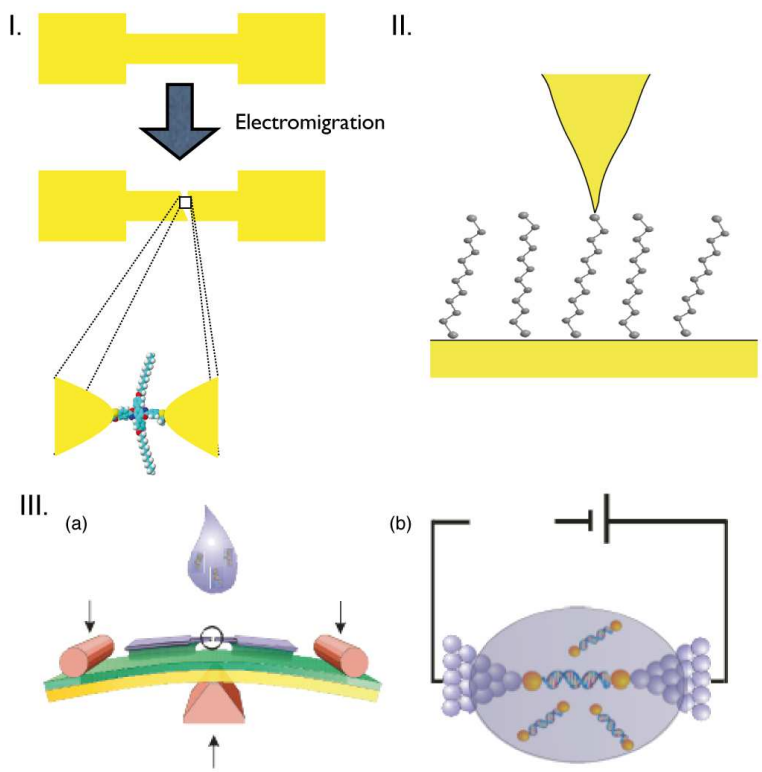

IV.
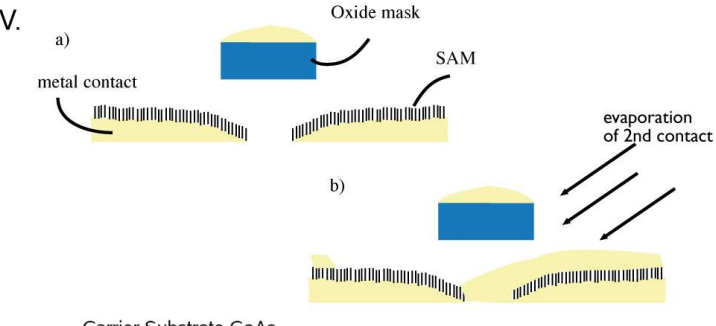

V.

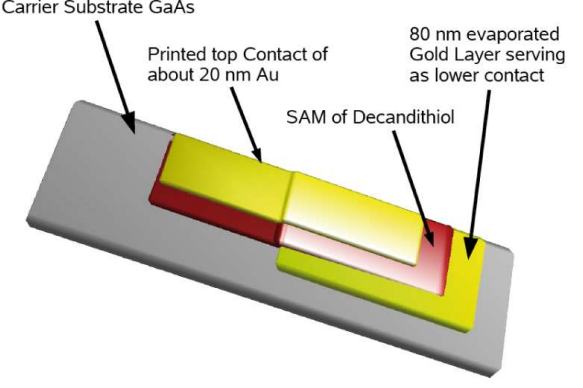

Fig. 4. Overview of contacting schemes. I. Electromigration; II. STM; III. MCBJ a) overview of the sample b) molecule in the center of the electrodes IV. Shadow mask evaporation a) formation of SAM on the initial contacts b) formation of second contact.
In the following, we want to discuss several contacting techniques which were used in the past decade to form metallic contacts to molecular entities. The techniques can be generally grouped in two categories, junctions aiming at single molecule contacts and those contacting ensembles of molecules. We will first discuss single molecule junctions and then show why studies of ensembles of molecules are necessary as well to understand the behavior of the molecular junctions. A summary of the contacting techniques is shown in Fig. 4.

\subsection{Electromigration}

When a large current is passed through a metallic wire, the atoms of this wire are rearranged due to the momentum transfer which happens during current transport [17]. Thin metallic wires can be completely disconnected using this technique, forming a nanometer-sized gap between the two sides of the break. Molecules are deposited on top of these gaps from solution [18]. Ideally, a molecular junction is formed by this process if a single molecule is situated between the two metallic electrodes. The formation of such a junction is a highly statistical process $[19,20]$. Therefore, a large number of junctions have to be measured and compared to junctions without molecules or with molecules without free electronic states (e.g. alkanes) in order to make definite statements on the electronic properties of the tested molecules. The main advantages of this technique are the stability during temperature changes and the possibility to fabricate a gate electrode comparably close to the molecular junctions. This is commonly realized by placing the junctions on a highly doped semiconductor substrate, which is isolated with respect to the metallic leads by a natural oxide. Thus the electronic states of the junctions can be tuned and, in connection with variations in temperature, the junctions can be fully electrically characterized.

The prevailing result in electromigrated junctions is the Coulomb blockade [19]. It can be said that CB appears with an increased probability if molecules with conjugated backbones are present in the junction. Using this technique, the vibrational spectra of oligophenylenevinylene (OPV) could be identified, indicating that the CB structure indeed stems from transport through the molecule [21]. Other molecular properties like the Kondo resonances, which occur due to unpaired spins coupling to the electrons in the metallic leads, were identified as well in $\mathrm{C}_{60}$ molecules [22] and OPV [23]. $\mathrm{C}_{60}$ molecules also showed the presence of mechanical resonances [24]. These resonances could be tuned by changing the distance between the electrodes [25]. This was made possible by etching the native oxide under the metallic electrodes slightly and subsequently bending the substrate. The technique then resembles the MCBJ technique (Sect. 4.2 ) with an additional gate but with a smaller range of mechanical motion.

\subsection{MCBJ technique}

Mechanically controllable breakjunctions (MCBJ) represent an established investigation tool for conductance 
measurements on molecules [5, 26-28]. This technique is based on the lithographic fabrication of a free suspended metal wire of about $100 \mathrm{~nm}$ in width on a substrate. The substrate is then carefully bent with a centered, vertical pushing rod, which can be moved with an accuracy in the micrometer range. By fixing the sides of the substrate with counter supports, the vertical pushing is translated into a horizontal stretching of the wire, with a translation factor of $10^{-4}$ to $10^{-5}$. Thus, the wire successively narrows down and finally tears apart, thereby ideally providing two atomically sharp electrodes facing each other at the two ends of the metal wire. The MCBJ technique provides an electrode gap, which can be tuned and stabilized with sub-angstrom precision. The gap is then bridged by applying molecules either in solution [29, 30] or by evaporation [31]. The molecules are chemically tailored with anchor groups that bind to metal surfaces, so the bridging of the gap is achieved by chemical self-assembly.

The high mechanical stability of the MCBJ technique allows for performing $I-V$ sweeps as well as for collecting statistical data by repeatedly opening and closing the junction. When the wire opens with sufficiently sharp tips, single molecule measurements can be performed. The exact atomic conformation of the electrodes, however, is unknown in this technique, because no current imaging technique can provide reliable information on the required scale.

When a constant bias voltage is applied, the current is measured while repeatedly opening and closing the junction. From these current vs. distance measurements, conductance histograms can be obtained by summing up every measured conductance value over all cycles. Goldgold junctions exhibit a prominent conductance peak at $1.0 G_{0}$, because this value corresponds to the conductance of a monoatomic gold chain. $G_{0}$ denotes the conductance quantum $2 e^{2} / h=77.5 \mu \mathrm{S}$, though the term "quantum" should not be taken literally: conductance is not quantized and can easily assume values below one $G_{0}$.

When the opening and closing of a MCBJ is performed in the presence of organic molecules, a change in the conductance as a function of electrode distance can be expected. Metallic contacts with a conductance above $1 G_{0}$ are generally not affected, but the behavior in the tunneling regime changes. The "simplest" molecule is the hydrogen molecule, $\mathrm{H}_{2}$. This molecule was evaporated into a $\mathrm{Pt}$ junction and the conductance histograms were investigated [26]. A clear conductance peak at $1 G_{0}$ arose, which is expected for the orbital structure of $\mathrm{H}_{2}$, but not for Pt. Additional analysis of $\mathrm{d} I / \mathrm{d} V$ curves confirmed the assumption that the current transport is dominated by the presence of $\mathrm{H}_{2}$.

If half a monolayer of $\mathrm{C}_{60}$ molecules is evaporated on the electrodes, an additional conductance peak arises at $0.1 G_{0}[28,31]$. The junctions were further characterized by measuring $\mathrm{d} I / \mathrm{d} V$ curves using a lock-in amplifier. In this measurement additional excitations of the system can be seen as maxima in the conductance of the junc- tions. Typical excitations are given by phonons. Conductance maxima could be found at voltages which correspond to typical vibration energies of the $\mathrm{C}_{60}$ molecule [28] and were found by other groups using a different technique as well [32]. Using the MCBJ setup, more complex molecules, which are chemisorbed on the metallic contacts, can be investigated as well [33, 34]. One special example is a molecular diode [35], which was developed based on the famous suggestion by Aviram and Ratner [2]. Experiments focused on $I-V$ measurements at a temperature of $30 \mathrm{~K}$, showing clearly asymmetric behavior.

\subsection{STM technique}

Scanning tunneling microscopes (STMs) have been widely used as a testbed for molecular electronics $[16,36,37]$. An STM consists of a conducting tip, which is ideally atomically sharp. The tip is brought into close proximity to the conducting sample by measuring the tunneling current between the tip and the sample. Any current changes can immediately be compensated by vertical adjustment of the probe. Thus, when scanning with this probe over a conducting surface, a height profile can be recorded by keeping the tunneling current constant. The drawback of this approach is that changes in conductivity cannot be easily distinguished from changes in height of the underlying structure. STMs may also be used similar to breakjunctions. Instead of scanning the surface, the tip is moved down and up again, thus making contact with the sample. If a metal sample is covered by a monolayer of molecules, the STM tip may bind to the upper end of one or a few molecules, thereby forming a metal-molecule-metal junction. The tip movement can be performed at high speed, thereby enabling the recording of thousands of opening and closing cycles with a single sample [37]. While doing statistics on the gap bridging cycle is a proper application of STMs in molecular electronics, performing $I-V$ sweeps proves to be difficult due to the poor mechanical stability of the tip.

Wandlowski et al. studied in detail the conductance of dissolved alkanedithiols in an STM setup and derived possible configurations of $\mathrm{Au}-$ molecule- $\mathrm{Au}$ junctions from conductance histograms [37]. Peaks in the histograms can be categorized in three series of low (L), medium $(\mathrm{M})$ and high $(\mathrm{H})$ conductance, respectively, each series consisting of up to four peaks. Each series is attributed to a different configuration of the investigated molecule. The $\mathrm{H}$ peaks correspond to a molecule having anchored its thiol ends between two gold atoms each, called "bridge" or "hollow" conformation. Docking the thiols atop of single gold atoms yields a poorer conductance, resulting in the $\mathrm{M}$ peaks. When the bond is instable or the molecule has structural defects, even lower conductance values are measured, giving rise to the $\mathrm{L}$ series. The first peak of each series corresponds to a single molecule contact. The subsequent peaks are located at multiples of the first peak, thus they are attributed to 
twice and triple molecular bridges, which occur less often statistically. The outcome of this experiment implies caution for future measurements. Whenever an experimental setup favors a specific junction conformation, the distribution of measured conductance values may strongly shift to one of the series described above.

Molecules that exhibit a planar structure show more complex conformation changes than simple alkanes. Venkataraman et al. investigated the influence of twisting on the conductance of molecular junctions [38]. Different types of biphenyls were studied, varying by their twist angle, which in turn is dominated by the choice of proper side groups. Theory predicts a decrease in conductance proportional to the square cosine of the twist angle [39]. For different biphenyls, thousands of opening/closing cycles were recorded and plotted in histograms. The histogram of every biphenyl exhibits a peak, which is attributed to a molecular junction. The peak actually shifts to lower conductance values with increasing twist angle of the biphenyl. A plot of each peak's conductance vs. the square cosine reveals the predicted linear dependence.

\subsection{Contacting ensembles of molecules}

Contacts on the angstrom scale, as they are necessary to contact single molecules, cannot be produced reliably with the contacting techniques described above. One way to determine the molecular properties of the junctions is to conduct a high number of experiments and to look for influences of the exact molecular structure on the statistical distribution of the characteristics. This is a rather time consuming procedure and systematic changes in some molecular properties (for example the endgroups) are difficult to detect. Another possibility therefore relies on the fabrication of large-area contacts to ensembles of molecules. The fabrication of such structures starts with the formation of a SAM on a bottom electrode, which can be either a metal or a highly conducting semiconductor. The second electrode is deposited on top of the SAM. Conduction is then measured between these two electrodes through the SAM.

The deposition of the top electrode poses the main challenge in this approach since no short-circuit between the two metals may be created in this step. One way to avoid this is to restrict the area of overlap by shadow evaporation to a few tens of $\mathrm{nm}^{2}[40,41]$. Typical $I-V$ characteristics of phenylenes and thiophenes, which were taken in this technique, exhibit activated transport with $E_{\text {act }}$ on the order of $100 \mathrm{meV}$. This is much lower than would be expected based on the HOMO-LUMO gap of these molecules, which is about $2 \mathrm{eV}$. The transport mechanism is most likely caused by interplay between the metal of the electrode and the SAM. Artefacts due to the evaporation of the metal on the SAM can be excluded, because $E_{\text {act }}$ changes when the contacting metal is changed, and is similar when the top or bottom metal changes [40].

It was also demonstrated that spinning of a conductive polymer (PEDOT:PSS) on top of the SAM, which is again formed on a metallic substrate, produces reliable contacts to the molecules on areas ranging from $100 \mathrm{~nm}^{2}$ to about $100 \mu \mathrm{m}^{2}$ [42]. This method was used to demonstrate pure tunneling through alkane monolayers. The $I-V$ characteristics were temperature independent and showed exponential decay of the conductance with increase in the molecular chain length. In addition to this, a molecular switch, which was opened and closed by irradiation of light, could be demonstrated successfully by this technique [43].

Deposition of the top electrode can be also done by using a soft lithographic step, which is called nano transfer printing (nTP). For this method gold is evaporated on top of a soft polymer (polydimethylsiloxane, PDMS), which can be cast into various shapes $[41,44]$. This metal is then brought into contact with a surface that is covered with a SAM of molecules with functional groups (thiol groups in most cases) on both ends. The upper functional groups then tear the gold off the PDMS and the top electrode is formed. Current is measured between the top electrode and the substrate, which can be either metallic or a highly doped semiconductor. It was shown that the presence of an alkane SAM on top of a GaAs substrate suppresses the Shottky barrier, which is usually present when a metal is brought into contact with a semiconductor [45]. Transport through alkane molecules shows a superposition of tunneling behavior and activated behavior through the molecules with $E_{\text {act }}=2 \mathrm{eV}$ [46]. Interestingly, in this measurement the tunneling current is temperature dependent, because the conformation of the alkane molecules changes with temperature and thus decreases the width of the tunneling barrier, as was found previously in STM measurements [47].

\section{Conclusions}

Several contacting techniques to single molecules and to molecular ensembles were presented. It is clear that no single contacting technique can provide the full information needed to fully understand transport properties of molecules. One of the main open questions is the role of the contacting end groups in the transport. Alternatives to the most common thiol end groups are currently investigated. To answer such open questions a combination of available contacting techniques is needed. a

\section{References}

[1] G.E. Moore, Electronics 38, 8 (1965).

[2] A. Aviram, M.A. Ratner, Chem. Phys. Lett. 29, 277 (1974).

[3] M.A. Reed, C. Zhou, C.J. Muller, T.P. Burgin, J.M. Tour, Science 278, 252 (1997).

[4] C. Zhou, M.R. Deshpande, M.A. Reed, L. Jones II, J.M. Tour, Appl. Phys. Lett. 71, 611 (1997).

[5] C. Kergueris, J.-P. Bourgoin, S. Palacin, D. Esteve, C. Urbina, M. Magoga, C. Joachim, Phys. Rev. B 59, 12505 (1999). 
[6] C.P. Collier, E.W. Wong, M. Belohradsk, F.M. Raymo, J.F. Stoddart, P.J. Kuekes, R.S. Williams, J.R. Heath, Science 285, 391 (1999).

[7] Introducing Molecular Electronics, Lecture Notes in Physics, Eds. G. Cuniberti, G. Fagas, K. Richter, Springer, Berlin 2005, p. 519.

[8] W. Wang, T. Lee, M.A. Reed, Phys. Rev. B 68 035416 (2003).

[9] R.F. Service, Science 302, 556 (2003).

[10] H.v. Houten, C.W.J. Beenakker, A.A.M. Stargin, Coulomb-Blockade Oscillations in Semiconductor Nanostructures, Plenum, New York 1992, B294.

[11] W. Wang, T. Lee, M.A. Reed, Phys. Rev. B 68, 035416 (2003).

[12] A. Nitzan, Annu. Rev. Phys. Chem. 52, 681 (2001).

[13] B.B. Schmidt, M.H. Hettler, G. Schoen, Phys. Rev. B 75, 115125 (2007)

[14] R.G. Nuzzo, D.L. Allara, J. Am. Chem. Soc. 105, 4481 (1983).

[15] C.W. Sheen, J.X. Shi, J. Maartensson, A.N. Parikh, D.L. Allara, J. Am. Chem. Soc. 114, 1514 (1992).

[16] L. Venkataraman, J.E. Klare, I.W. Tam, C. Nuckolls, M.S. Hybertsen, M.L. Steigerwald, Nano Lett. 6, 458 (2006).

[17] H. Park, A.K.L. Lim, A.P. Alivisatos, J. Park, P.L. McEuen, Appl. Phys. Lett. 75, 301 (1999).

[18] E.A. Osorio, K. O'Neill, N. Stuhr-Hansen, O.F. Nielsen, T. Bjornholm, H.S.J. van der Zant, Adv. Mater. 19, 281 (2007).

[19] A. de Picciotto, J.E. Klare, C. Nuckolls, K. Baldwin, A. Erbe, R. Willett, Nanotechnology 16, 3110 (2005).

[20] H.S.J. van der Zant, Y. Kervennic, M. Poot, K. O'Neill, Z. de Groot, J.M. Thijssen, H.B. Heersche, N. Stuhr-Hansen, T. Bjornholm, D. Vanmaekelbergh, C.A. van Walree, L.W. Jenneskens, Faraday Discuss. 131, 347 (2006).

[21] H.S.J. van der Zant, E.A. Osorio, M. Poot, K. O'Neill, Phys. Status Solidi B 243, 3408 (2006).

[22] D. Natelson, L.H. Yu, J.W. Ciszek, Z.K. Keane, J.M. Tour, Chem. Phys. 324, 267 (2006).

[23] E.A. Osorio, K. O'Neill, M. Wegewijs, N. StuhrHansen, J. Paaske, T. Bjornholm, H.S.J. van der Zant, Nano Lett. 7, 3336 (2007).

[24] H. Park, J. Park, A.K.L. Lim, E.H. Anderson, A.P. Alivisatos, P.L. McEuen, Nature 407, 57 (2001).

[25] J.J. Parks, A.R. Champagne, G.R. Hutchison, S. Flores-Torres, H.D. Abruna, D.C. Ralph, Phys. Rev. Lett. 99, 026601 (2007).

[26] R.H.M. Smit, Y. Noat, C. Untiedt, N.D. Lang, M.C. Hemert, J.M. Ruitenbeek, Nature 419, 906 (2002).
[27] J. Reichert, R. Ochs, D. Beckmann, H.B. Weber, M. Mayor, H. v. Löhneysen, Phys. Rev. Lett. 88, 176804 (2002).

[28] T. Bohler, A. Edtbauer, E. Scheer, Phys. Rev. B 76, 125432 (2007).

[29] J. Reichert, R. Ochs, D. Beckmann, H.B. Weber, M. Mayor, H.v. Löhneysen, Phys. Rev. Lett. 88, 176804 (2002).

[30] R. Huber, M. T. Gonzalez, S. Wu, M. Langer, S. Grunder, V. Horhoiu, M. Mayor, M.R. Bryce, C. Wang, R. Jitchati, Ch. Schoenenberger, M. Calame, J. Am. Chem. Soc. 130, 1080 (2008).

[31] T. Bohler, J. Grebing, A. Mayer-Gindner, H.V. Löhneysen, E. Scheer, Nanotechnology 15, S465 (2004).

[32] H. Park, J. Park, A.K.L. Lim, E.H. Anderson, A.P. Alivisatos, P.L. McEuen, Nature 407, 57 (2001).

[33] J. Reichert, H.B. Weber, M. Mayor, H. v. Löhneysen, Appl. Phys. Lett. 82, 4137 (2003).

[34] N. Kang, A. Erbe, E. Scheer, to be published.

[35] M. Elbing, R. Ochs, M. Koentopp, M. Fischer, C. v. Hänisch, F. Weigend, F. Evers, H.B. Weber, M. Mayor, Proc. Natl. Acad. Sci. 102, 8815 (2005).

[36] X.Y. Xiao, B.Q. Xu, N.J. Tao, Nano Lett. 4, 267 (2004).

[37] C. Li, I. Pobelov, T. Wandlowski, A. Bagrets, A. Arnold, F. Evers, J. Am. Chem. Soc. 130, 318 (2008).

[38] L. Venkataraman, J.E. Klare, C. Nuckolls, M.S. Hybertsen, M.L. Steigerwald, Nature 442, 904 (2006).

[39] S. Woitellier, J.P. Launay, C. Joachim, Chem. Phys. 131, 481 (1989).

[40] N.B. Zhitenev, A. Erbe, Z. Bao, Phys. Rev. Lett. 92, 186805 (2004).

[41] A. Erbe, W. Jiang, Z. Bao, D. Abusch-Magder, D.M. Tennant, E. Garfunkel, N. Zhitenev, J. Vac. Sci. Technol. B 23, 3132 (2005).

[42] H.B. Akkerman, P.W. M. Blom, D.M. de Leeuw, B. de Boer, Nature 441, 69 (2006).

[43] A.J. Kronemeijer, H.B. Akkerman, T. Kudernac, B.J. van Wees, B.L. Feringa, P.W.M. Blom, B. de Boer, Adv. Mater. 20, 1467 (2008).

[44] Y.L. Loo, R.L. Willett, K.W. Baldwin, J.A. Rogers, Appl. Phys. Lett. 81, 562 (2002).

[45] Y.L. Loo, D.V. Lang, J.A. Rogers, J.W.P. Hsu, Nano Lett. 3, 913 (2003).

[46] C. Kreuter, S. Bächle, E. Scheer, A. Erbe, unpublished results.

[47] W. Haiss, H. v. Zalinge, D. Bethell, J. Ulstrup, D.J. Schiffrin, R.J. Nichols, Faraday Discuss. 131, 253 (2006). 